\title{
Sign-Changing Solutions for a Fourth-Order Elliptic Equation with Hardy Singular Terms
}

\author{
Ruichang Pei ${ }^{1,2}$ and Jihui Zhang ${ }^{1}$ \\ ${ }^{1}$ Institute of Mathematics, School of Mathematics and Computer Sciences, Nanjing Normal University, Nanjing 210097, China \\ ${ }^{2}$ School of Mathematics and Statistics, Tianshui Normal University, Tianshui 741001, China \\ Correspondence should be addressed to Ruichang Pei; prc211@163.com
}

Received 8 August 2013; Accepted 28 October 2013

Academic Editor: Wan-Tong Li

Copyright (c) 2013 R. Pei and J. Zhang. This is an open access article distributed under the Creative Commons Attribution License, which permits unrestricted use, distribution, and reproduction in any medium, provided the original work is properly cited.

The existence and multiplicity of sign-changing solutions for a class of fourth elliptic equations with Hardy singular terms are established by using the minimax methods.

\section{Introduction}

Consider the following Navier boundary value problem:

$$
\begin{gathered}
\triangle^{2} u(x)-\frac{N^{2}(N-4)^{2}}{16} \frac{u}{|x|^{4}}=f(x, u), \text { in } \Omega, \\
u=\Delta u=0 \text { on } \partial \Omega,
\end{gathered}
$$

where $\Omega$ is a bounded smooth domain in $\mathbb{R}^{N}(N \geq 5), 0 \in \Omega$.

The conditions imposed on $f(x, t)$ are as follows:

$\left(H_{1}\right)$ there exists $C>0$ such that

$$
|f(x, t)| \leq C\left(1+|t|^{p}\right), \forall t \in \mathbb{R}, \quad \forall x \in \Omega,
$$

where $1<p<(N+4) /(N-4)$;

$\left(H_{2}\right) f \in C(\bar{\Omega} \times \mathbb{R}, \mathbb{R}), \quad f(x, t) t \geq 0$ for all $x \in \Omega, t \in \mathbb{R} ;$

$\left(H_{3}\right) \lim _{|t| \rightarrow 0} f(x, t) / t=f_{0}, \lim _{|t| \rightarrow \infty} f(x, t) / t=l$ uniformly for $x \in \Omega$, where $f_{0}$ and $l$ are constants;

$\left(H_{4}\right) \lim _{|t| \rightarrow \infty}[f(x, t) t-2 F(x, t)]=-\infty$ uniformly for $x \epsilon$ $\Omega$, where $F(x, t)=\int_{0}^{t} f(x, s) d s ;$

$\left(H_{5}\right)$ there exist $\mu>2$ and $R>0$ such that

$$
0<\mu F(x, t) \leq f(x, t) t, x \in \Omega, \quad|t| \geq R
$$

$\left(H_{6}\right) f(x, t)$ is odd in $t$.
In recent years, this fourth-order semilinear elliptic problem:

$$
\begin{gathered}
\triangle^{2} u(x)+c \Delta u=f(x, u), \quad \text { in } \Omega, \\
u=\Delta u=0 \quad \text { on } \partial \Omega,
\end{gathered}
$$

can be considered as an analogue of a class of second-order problems which have been studied by many authors. In [1], there was a survey of results obtained in this direction. In [2], Micheletti and Pistoia showed that (4) admits at least two solutions by a variation of linking if $f(x, u)$ is sublinear. And in [3], the authors proved that the problem (4) has at least three solutions by a variational reduction method and a degree argument. In [4], Zhang and Li showed that (4) admits at least two nontrivial solutions by Morse theory and local linking if $f(x, u)$ is superlinear and subcritical on $u$.

To the authors' knowledge, there seem few results about the sign-changing solutions on problem (1) with hardy singular terms. In this paper, motivated by [5-8], the existence and multiplicity of sign-changing solutions for problem (1) are obtained by introducing a compact embedding theorem and a maximum principle. Our results are new.

\section{Preliminaries and Auxiliary Lemmas}

We introduce the new working space $E$ which is obtained by the completion of $C_{0}^{\infty}(\Omega)$ with respect to the norm (see [5])

$$
\|u\|=\left(\int_{\Omega}\left(|\Delta u|^{2}-\frac{N^{2}(N-4)^{2}}{16} \frac{|u|^{2}}{|x|^{4}}\right) d x\right)^{1 / 2}
$$


associated with the inner product

$$
\langle u, v\rangle=\int_{\Omega}\left(\Delta u \Delta v-\frac{N^{2}(N-4)^{2}}{16} \frac{u v}{|x|^{4}}\right) d x .
$$
norm.

Throughout this paper, we denoted by $\|\cdot\|_{p}$ the $L^{p}(\Omega)$

At first, we here give two important lemmas.

Lemma 1. $E \hookrightarrow \hookrightarrow L^{2}(\Omega)($ see $[5])$.

Lemma 2 (see $\left[6\right.$, Corollary 4.1]). Assume $N \geq 5, V \in L^{\infty}(\Omega)$, and $V \geq 0$. Let us suppose that the operator $\triangle^{2}-\left(V /|x|^{4}\right)$ is coercive on $H^{2}(\Omega) \cap H_{0}^{1}(\Omega)$. Let $f \in L^{2}(\Omega)$ such that $f \geq 0$. Let $u \in H^{2}(\Omega)$ be a solution of

$$
\begin{gathered}
\triangle^{2} u(x)-\frac{V}{|x|^{4}} u=f, \text { in } \Omega, \\
u=\triangle u=0 \text { on } \partial \Omega .
\end{gathered}
$$

Then $u \geq 0$ in $\Omega$.

Now, we consider the following eigenvalue problem:

$$
\begin{gathered}
\triangle^{2} u(x)-\frac{N^{2}(N-4)^{2}}{16|x|^{4}} u=\lambda u, \text { in } \Omega, \\
u=\Delta u=0 \text { on } \partial \Omega .
\end{gathered}
$$

The first eigenvalue of this problem is given by

$$
\lambda_{1}=\inf \left\{\|u\|^{2}: u \in E,\|u\|_{2}=1\right\} .
$$

By Lemma 1, $E \hookrightarrow W^{1, p}(\Omega) \hookrightarrow \hookrightarrow L^{2}(\Omega)$ for $p \rightarrow 2^{-}$. The minimizing sequence is compact in $L^{2}(\Omega)$. By standard argument, we may assume that the first eigenfunction $\phi_{1}$ is positive in $\Omega$ (see [9, page 167]). The second eigenvalue is given by

$$
\lambda_{2}=\inf \left\{\|u\|^{2}: u \in E, \int_{\Omega} u \phi_{1}=0,\|u\|_{2}=1\right\}
$$

which possesses a sign-changing eigenfunction $\phi_{2}$. Similarly, we can characterize the $n$th eigenvalue $\lambda_{n}$ with a signchanging eigenfunction. By standard elliptic theory, $\lambda_{n} \rightarrow$ $\infty$ as $n \rightarrow \infty$.

It follows from $\left(H_{1}\right)$ that the functional

$$
\begin{aligned}
I(u)= & \frac{1}{2} \int_{\Omega}|\Delta u|^{2} d x-\frac{N^{2}(N-4)^{2}}{32} \\
& \times \int_{\Omega} \frac{u^{2}}{|x|^{4}}-\int_{\Omega} F(x, u) d x
\end{aligned}
$$

is of $C^{1}$ on the space $E$. Under the condition $\left(H_{1}\right)$, the critical points of $I$ are solutions of problem (1).

If $l$ in the above condition $\left(H_{3}\right)$ is an eigenvalue of $\left(\triangle^{2}-\right.$ $\left.\left(N^{2}(N-4)^{2} / 16\right)\left(1 /|x|^{4}\right), E\right)$, then the problem (1) is called resonance at infinity. Otherwise, we call it nonresonance.

For looking for sign-changing solutions of problem (1), we recall a very useful result.
Proposition 3 (see [10, Theorem 3.2]). Let $X$ be a Hilbert space and $f$ be a $C^{1}$ functional defined on $X$. Assume that $f$ satisfies the (PS) condition on $X$ and $f^{\prime}(u)$ has the expression $f^{\prime}(u)=u-A u$ for $u \in X$. Assume that $D_{1}$ and $D_{2}$ are open convex subset of $X$ with the properties that $D_{1} \cap D_{2} \neq \emptyset$, $A\left(\partial D_{1}\right) \subset D_{1}$, and $A\left(\partial D_{2}\right) \subset D_{2}$. If there exists a path $h:[0,1] \rightarrow X$ such that

$$
\begin{gathered}
h(0) \in D_{1} \backslash D_{2}, \quad h(1) \in D_{2} \backslash D_{1}, \\
\underset{u \in \overline{D_{1}} \cap \overline{D_{2}}}{\inf } f(u)>\sup _{t \in[0,1]} f(h(t)),
\end{gathered}
$$

then $f$ has at least four critical points, one in $D_{1} \cap D_{2}$, one in $D_{1} \backslash \overline{D_{2}}$, one in $D_{2} \backslash \overline{D_{1}}$, and one in $X \backslash\left(\overline{D_{1}} \cup \overline{D_{2}}\right)$.

Remark 4. If $f$ satisfies the $(C)_{c}$ condition, then this proposition still holds (see [11]).

\section{Main Results}

Let us now state the main results.

Theorem 5. Assume conditions $\left(\mathrm{H}_{2}\right)$ and $\left(\mathrm{H}_{3}\right)$ hold. If $f_{0}<\lambda_{1}$ and $l \in\left(\lambda_{k}, \lambda_{k+1}\right)$ for some $k>2$, then problem (1) has a positive solution, a negative solution, and a sign-changing solution.

Remark 6. This result is similar to [7, Theorem 1.1]. As far as verifying the (PS) condition is concerned, our method is more simple than that in $[12,13]$.

Theorem 7. Assume conditions $\left(H_{2}\right)-\left(H_{4}\right)$ hold. If $f_{0}<\lambda_{1}$ and $l=\lambda_{k}$ for some $k>2$, then problem (1) has a positive solution, a negative solution, and a sign-changing solution.

Remark 8. When $l=\lambda_{k}(k>2)$, the case is called resonance and not considered by [7]. This result is completely new.

Theorem 9. Assume conditions $\left(H_{1}\right),\left(H_{5}\right)$, and $\left(H_{6}\right)$ hold. If $f_{0}=0$, then problem (1) has infinitely many sign-changing solutions.

Lemma 10. Under the assumptions of Theorem 5, if $\lambda_{k}<l<$ $\lambda_{k+1}$, then I satisfies the (PS) condition.

Proof. Let $\left\{u_{n}\right\} \subset E$ be a sequence such that $\left|I\left(u_{n}\right)\right| \leq c,<$ $I^{\prime}\left(u_{n}\right)$, and $\phi>\rightarrow 0$. Since

$$
\begin{aligned}
& \left\langle I^{\prime}\left(u_{n}\right), \phi\right\rangle \\
& =\int_{\Omega}\left(\Delta u_{n} \Delta \phi-\frac{N^{2}(N-4)^{2}}{16} \frac{u_{n} \phi}{|x|^{4}}\right) d x \\
& -\int_{\Omega} f\left(x, u_{n}\right) \phi d x=o(\|\phi\|)
\end{aligned}
$$

for all $\phi \in E$. If $\left\|u_{n}\right\|_{2}$ is bounded, we can take $\phi=u_{n}$. By $\left(H_{3}\right)$, there exists a constant $c>0$ such that $\left|f\left(x, u_{n}(x)\right)\right| \leq c\left|u_{n}(x)\right|$, a.e. $x \in \Omega$. So $u_{n}$ is bounded in $E$. If $\left\|u_{n}\right\|_{2} \rightarrow+\infty$, as $n \rightarrow$ $\infty$, set $v_{n}=u_{n} /\left\|u_{n}\right\|_{2}$, then $\left\|v_{n}\right\|_{2}=1$. Taking $\phi=v_{n}$ in (14), 
it follows that $\left\|v_{n}\right\|$ is bounded. Without loss of generality, we assume $v_{n} \rightarrow v$ in $E$, and then $v_{n} \rightarrow v$ in $L^{2}(\Omega)$. Hence, $v_{n} \rightarrow v$ a.e. in $\Omega$ and $\left|v_{n}\right| \leq q(x)\left(q(x) \in L^{2}(\Omega)\right)$. Dividing both sides of (14) by $\left\|u_{n}\right\|_{2}$, for all $\phi \in E$, we get

$$
\begin{gathered}
\int_{\Omega}\left(\triangle v_{n} \Delta \phi-\frac{N^{2}(N-4)^{2}}{16} \frac{v_{n} \phi}{|x|^{4}}\right) d x \\
-\int_{\Omega} \frac{f\left(x, u_{n}\right)}{\left\|u_{n}\right\|_{2}} \phi d x=o\left(\frac{\|\phi\|}{\left\|u_{n}\right\|_{2}}\right) .
\end{gathered}
$$

Then for a.e. $x \in \Omega$, we have $f\left(x, u_{n}\right) /\left\|u_{n}\right\|_{2} \rightarrow l v$ as $n \rightarrow \infty$. In fact, if $v(x) \neq 0$, by $\left(H_{3}\right)$, we have

$$
\begin{gathered}
\left|u_{n}(x)\right|=\left|v_{n}(x)\right|\left\|u_{n}\right\|_{2} \longrightarrow+\infty, \\
\frac{f\left(x, u_{n}\right)}{\left\|u_{n}\right\|_{2}}=\frac{f\left(x, u_{n}\right)}{u_{n}} v_{n} \longrightarrow l v .
\end{gathered}
$$

If $v(x)=0$, we have

$$
\frac{\left|f\left(x, u_{n}\right)\right|}{\left\|u_{n}\right\|_{2}} \leq c\left|v_{n}\right| \longrightarrow 0
$$

Since $\left|f\left(x, u_{n}\right)\right| /\left\|u_{n}\right\|_{2} \leq c\left|v_{n}\right| \leq c q(x)$, by (15) and the Lebesgue dominated convergence theorem, we arrive at

$$
\int_{\Omega} \Delta v \triangle \phi d x-\frac{N^{2}(N-4)^{2}}{16} \frac{v \phi}{|x|^{4}}-\int_{\Omega} l v \phi d x=0, \forall \phi \in E .
$$

It is easy to see that $v \not \equiv 0$. In fact, if $v \equiv 0$, then $\|v\|_{2}=0$ contradicts $\lim _{n \rightarrow \infty}\left\|v_{n}\right\|_{2}=\|v\|_{2}=1$. Hence, $l$ is an eigenvalue of $\left(\triangle^{2}-\left(N^{2}(N-4)^{2} / 16\right)\left(1 /|x|^{4}\right), E\right)$. This contradicts our assumption. Thus $\left\{u_{n}\right\}$ is bounded. By standard argument (see the proof of our Lemma 12 below), $\left\{u_{n}\right\} \rightarrow u$ in $E$. The lemma is proved.

Lemma 11. Under the assumptions of Theorem 7, if $l=\lambda_{k}$, then the functional I satisfies the $(C)$ condition which is stated in [11].

Proof. Suppose I satisfies

$$
\begin{array}{r}
I\left(u_{n}\right) \longrightarrow c \in \mathbb{R}, \quad\left(1+\left\|u_{n}\right\|\right)\left\|I^{\prime}\left(u_{n}\right)\right\| \\
\text { as } n \longrightarrow 0 \\
\longrightarrow \infty .
\end{array}
$$

In view of $\left(H_{3}\right)$, it suffices to prove that $u_{n}$ is bounded in $E$. Similar to the proof of Lemma 10, we have

$$
\begin{aligned}
& \int_{\Omega}\left(\Delta v \Delta \phi-\frac{N^{2}(N-4)^{2}}{16} \frac{v \phi}{|x|^{4}}\right) d x \\
& -\int_{\Omega} l v \phi d x=0, \forall \phi \in E .
\end{aligned}
$$

Therefore $v \neq \equiv$ is an eigenfunction of $\lambda_{k}$, and then $\left|u_{n}(x)\right| \rightarrow \infty$ for a.e. $x \in \Omega$. It follows from $\left(H_{4}\right)$ that

$$
\lim _{n \rightarrow+\infty}\left[f\left(x, u_{n}(x)\right) u_{n}(x)-2 F\left(x, u_{n}(x)\right)\right]=-\infty
$$

holds uniformly in $x \in \Omega$, which implies that

$$
\int_{\Omega}\left(f\left(x, u_{n}\right) u_{n}-2 F\left(x, u_{n}\right)\right) d x \longrightarrow-\infty \text { as } n \longrightarrow \infty .
$$

On the other hand, (19) implies that

$$
2 I\left(u_{n}\right)-\left\langle I^{\prime}\left(u_{n}\right), u_{n}\right\rangle \longrightarrow 2 c \text { as } n \longrightarrow \infty .
$$

Thus

$$
\int_{\Omega}\left(f\left(x, u_{n}\right) u_{n}-2 F\left(x, u_{n}\right)\right) d x \longrightarrow 2 c \text { as } n \longrightarrow \infty,
$$

which contradicts (22). Hence $u_{n}$ is bounded.

Lemma 12. Assume $\left(H_{1}\right)$ and $\left(H_{5}\right)$ hold. Then I satisfies the (PS) condition.

Proof. Assume that $\left\{u_{n}\right\}$ is a (PS) sequence; $\left\|I^{\prime}\left(u_{n}\right)\right\| \rightarrow 0$ and $\left\{I\left(u_{n}\right)\right\}$ is bounded. A routine argument implies that $\left\{\left\|u_{n}\right\|\right\}$ is bounded. By [6, Theorem A.2], we have

$$
E \hookrightarrow W_{0}^{1, q}(\Omega)
$$

where $1 \leq q<2$. For $p$ given in $\left(H_{1}\right), p<(n+4) /(n-4)$, and we may choose $q$ such that $(p+1)<q N /(N-q), q<2$. By the Sobolev embedding theorem, we have

$$
W^{1, q}(\Omega) \hookrightarrow \hookrightarrow L^{t}(\Omega), \forall t<\frac{N q}{N-q} .
$$

We infer from (26) that $\left\{u_{n}\right\}$ is compact in $L^{p+1}(\Omega)$. By $\left(H_{1}\right)$,

$$
\begin{aligned}
& \left\|u_{n}-u_{m}\right\|^{2} \\
& \quad=\int_{\Omega}\left|f\left(x, u_{n}\right)-f\left(x, u_{m}\right)\right|\left|u_{n}-u_{m}\right| d x+o(1) \\
& \quad \leq C\left(\int_{\Omega}\left|u_{n}-u_{m}\right|^{p+1} d x\right)^{1 /(p+1)}+o(1) \longrightarrow 0
\end{aligned}
$$

This completes the proof of this lemma.

For the aim of using Proposition 3 that proves our main results, we prove an important lemma below.

From previous Section 1, we know that $I$ is $C^{1}$ functional and its gradient at $u$ is given by

$$
\begin{gathered}
I^{\prime}(u)=u-A(u), A: E \rightarrow E \\
A(u)=\left(\triangle^{2}-\frac{N^{2}(N-4)^{2}}{16} \frac{1}{|x|^{4}}\right)^{-1} f(x, u) .
\end{gathered}
$$

Then $\langle A(u), \phi\rangle=\int_{\Omega} f(x, u) \phi d x$ for all $\phi \in E$. We consider the convex cones $P=\{u \in H: u \geq 0\}$ and $-P=\{u \in E: u \leq 0\}$; moreover, for $\epsilon>0$, assume

$$
\begin{aligned}
P_{\epsilon} & =\{u \in E: \operatorname{dist}(u, P)<\epsilon\}, \\
-P_{\epsilon} & =\{u \in E: \operatorname{dist}(u,-P)<\epsilon\} .
\end{aligned}
$$

Note that $P_{\epsilon}$ and $-P_{\epsilon}$ are open convex subsets of $E$ and $E \backslash\left(\overline{P_{\epsilon}} \cup\left(\overline{-P_{\epsilon}}\right)\right)$ contains only sign-changing functions. 
Lemma 13. Assume $\left(\mathrm{H}_{2}\right)$ and $\left(\mathrm{H}_{3}\right)$ hold. Then, there exists $\epsilon_{0}>0$ such that for $0<\epsilon \leq \epsilon_{0}$ there holds

$$
A\left(\partial\left( \pm P_{\epsilon}\right)\right) \subset \pm P_{\epsilon} .
$$

Moreover, if $u \in \pm P_{\epsilon}$ is a nontrivial solution of problem (1), then $u$ is positive (negative) in the sense that $u>0 \quad(u<$ $0)$ in $\Omega$.

Proof. Indeed, if $u \in E$ and $u^{+}=\max \{u, 0\}, u^{-}=\min \{u, 0\}$, then

$$
\begin{aligned}
\operatorname{dist}(A(u), P) & \leq \inf _{w \in P}\|A(u)-w\| \\
& =\inf _{w \in P}\left\|A(u)^{+}+A(u)^{-}-w\right\| \leq\left\|A(u)^{-}\right\| .
\end{aligned}
$$

For every $s \in(2,2 N /(N-4))$, there exists $C_{s}>0$ such that

$$
\left\|u^{ \pm}\right\|_{s} \leq \inf _{w \in \mp P}\|u-w\|_{s} \leq C_{s} \operatorname{dist}(u, \mp P) .
$$

Choose $\epsilon^{\prime}>0$ such that $\left(f_{0}+\epsilon^{\prime}\right)<\lambda_{1}$. Using (32), the Hölder inequality, the Poincaré inequality, and the Sobolev embedding theorem, we have

$$
\begin{aligned}
& \operatorname{dist}(A(u), P)\left\|A(u)^{-}\right\| \\
& \leq\left\|A(u)^{-}\right\|^{2} \\
& =\int_{\Omega} f(x, u) A(u)^{-} d x \\
& \leq \int_{\Omega} f\left(x, u^{-}\right) A(u)^{-} d x \\
& \leq \int_{\Omega}\left(\left(f_{0}+\epsilon^{\prime}\right)\left|u^{-}\right|+C_{\epsilon}^{\prime}\left|u^{-}\right|^{p}\right) A(u)^{-} d x \\
& \leq\left(f_{0}+\epsilon^{\prime}\right)\left\|u^{-}\right\|_{2}\left\|A(u)^{-}\right\|_{2} \\
& +C_{\epsilon}^{\prime}\left\|u^{-}\right\|_{p+1}^{p}\left\|A(u)^{-}\right\|_{p+1} \\
& \leq\left(f_{0}+\epsilon^{\prime}\right) \inf _{w \in P}\|u-w\|_{2}\left\|A(u)^{-}\right\|_{2} \\
& +C \inf _{w \in P}\|u-w\|_{p+1}^{p}\left\|A(u)^{-}\right\|_{p+1} \\
& \leq \frac{f_{0}+\epsilon^{\prime}}{\lambda_{1}} \operatorname{dist}(u, P)\left\|A(u)^{-}\right\| \\
& +C \operatorname{dist}_{(u, P)^{p}\left\|A(u)^{-}\right\|,}
\end{aligned}
$$

where $C_{\epsilon}^{\prime}, C>0$ are constants. Hence

$$
\operatorname{dist}(A(u), P) \leq\left(\delta+C \operatorname{dist}(u, P)^{p-1}\right) \operatorname{dist}(u, P),
$$

where $\delta=\left(f_{0}+\epsilon^{\prime}\right) / \lambda_{1}<1$. Take $\epsilon_{0}$ such that $\delta_{1}=\delta+$ $C \epsilon_{0}^{p-1}<1$. Now if $\operatorname{dist}(u, P)<\epsilon<\epsilon_{0}$, then we have

$$
\operatorname{dist}(A(u), P) \leq \delta_{1} \operatorname{dist}(u, P) \text {. }
$$

Thus for every $u \in \partial P_{\epsilon}$, by (35) we have

$$
\operatorname{dist}(A(u), P) \leq \delta_{1} \epsilon \text {; }
$$

thus $A(u) \in P_{\epsilon}$. Hence $A\left(\partial P_{\epsilon}\right) \subset P_{\epsilon}$. In a similar way, $A\left(\partial\left(-P_{\epsilon}\right)\right) \subset\left(-P_{\epsilon}\right)$. If $0<\epsilon \leq \epsilon_{0}$, and $u \in P_{\epsilon}$ (resp., $\left.-P_{\epsilon}\right)$ is a nontrivial solution of problem $(1)$, then $I^{\prime}(u)=0$. By (35) we have $\operatorname{dist}(u, P)=0$; that is, $u \in P$ (resp., $u \in-P$ ). By Lemma 2, we imply that $u>0 \quad(u<0)$ in $\Omega$.

Lemma 14. Assume $\left(H_{1}\right),\left(H_{2}\right)$, and $\left(H_{5}\right)$ hold. Then, there exists $\epsilon_{0}>0$ such that for $0<\epsilon \leq \epsilon_{0}$ there holds

$$
A\left(\partial\left( \pm P_{\epsilon}\right)\right) \subset \pm P_{\epsilon}
$$

Proof. The proof is quite similar to that of Lemma 4.2 in [8]. We omit it here.

Lemma 15. Assume $\left(H_{5}\right)$ holds. Then

$$
I(u) \longrightarrow-\infty, \forall u \in E_{k},
$$

where the definition of $E_{k}$ introduced in our proof of Theorem 9. Proof. Because $\operatorname{dim} E_{k}<\infty$, then by $\left(H_{5}\right)$,

$$
\frac{I(u)}{\|u\|^{2}} \leq \frac{1}{2}-\int_{\Omega} \frac{F(x, u)}{\|u\|^{2}} d x \longrightarrow-\infty
$$

as $\|u\| \rightarrow \infty, u \in E_{k}$. This lemma follows immediately.

Lemma 16. Assume $\left(\mathrm{H}_{2}\right)$ and $\left(\mathrm{H}_{3}\right)$ hold. Let $0<\epsilon \leq \epsilon_{0}$, and then there exists $C_{0}>-\infty$ such that inf $\overline{\overline{P_{\epsilon}} \cap\left(-\overline{P_{\epsilon}}\right)} I(u)=C_{0}$.

Proof. By the conditions $\left(\mathrm{H}_{2}\right)$ and $\left(\mathrm{H}_{3}\right)$, we know that, for any $\epsilon^{\prime}>0$, there exists $C>0$, such that

$$
|f(x, t)| \leq\left(f_{0}+\epsilon^{\prime}\right)|t|+C|t|^{p} \quad\left(1<p<\frac{N+4}{N-4}\right) .
$$

Using (40) and the Sobolev embedding theorem, we have

$$
\begin{aligned}
I(u) & =\frac{1}{2}\|u\|^{2}-\int_{\Omega} F(x, u) d x \\
& \geq \frac{1}{2}\|u\|^{2}-\frac{1}{2}\left(f_{0}+\epsilon^{\prime}\right) \int_{\Omega} u^{2} d x-C\|u\|_{p+1}^{p+1} \\
& \geq-\frac{1}{2}\left(f_{0}+\epsilon^{\prime}\right)\|u\|_{2}^{2}-C\|u\|_{p+1}^{p+1} .
\end{aligned}
$$

By (32) we have $\left\|u^{ \pm}\right\|_{s} \leq C_{s} \epsilon_{0}$ for every $u \in P_{\epsilon} \cap\left(-P_{\epsilon}\right)$. So there exists $C_{0}>-\infty$ such that

$$
\inf _{\overline{P_{\epsilon}} \cap\left(-\overline{P_{\epsilon}}\right)} I(u)=C_{0} .
$$

Hence this lemma is proved. 


\section{Proof of the Main Results}

Proof of Theorem 5 and Theorem 7. Motivated by the Proof of Theorem 4.2 in [10], we still define a path $h_{R}:[0,1] \rightarrow E$ as

$$
h_{R}(t)=R \phi_{1} \cos \pi t+R \phi_{2} \sin \pi t, 0 \leq t \leq 1 .
$$

Obviously, $h_{R}(0) \in P_{\epsilon} \backslash\left(-P_{\epsilon}\right)$ and $h_{R}(1) \in\left(-P_{\epsilon}\right) \backslash P_{\epsilon}$. By the Fatou's lemma, the condition $\left(H_{3}\right)$ with $l>\lambda_{2}$ and a direct computation shows that

$$
\lim _{R \rightarrow+\infty} \sup _{t \in[0,1]} I\left(h_{R}(t)\right)=-\infty
$$

So, it yields that there exists $R_{0}$ such that $I\left(h_{R_{0}}(t)\right)<C_{0}-$ $c^{*}\left(c^{*}>0\right)$. Hence we obtain

$$
\inf _{\overline{P_{\epsilon}} \cap\left(-\overline{P_{\epsilon}}\right)} I(u)>\sup _{t \in[0,1]} I(h(t)) .
$$

By using Lemmas 10, 11, and 13, Proposition 3, and Lemma 16, we can find a critical point in $P_{\epsilon} \backslash\left(-\overline{P_{\epsilon}}\right)$ which is a positive solution, a critical point in $\left(-P_{\epsilon}\right) \backslash \overline{P_{\epsilon}}$ which is a negative solution, and a critical point in $E \backslash\left(\overline{P_{\epsilon}} \cup\left(-\overline{P_{\epsilon}}\right)\right)$ which is a sign-changing solution.

Before beginning our proof of Theorem 9, we need the following important proposition.

Proposition 17 (see [9, Theorem 5.6]). Assume E is a Hilbert space with inner product $<,>$ and the corresponding norm $\|\cdot\|, I \in C^{1}(E, R)$ and $I(u)=(1 / 2)\|u\|^{2}-G(u), u \in E$, where $G \in C^{1}(E, R)$. P denotes a positive closed convex cone of $E$.

(A) Assume that $A\left( \pm D_{0}\right) \subset \pm D_{0}$, where $D_{0}:=\{u \in E$ : $\left.\operatorname{dist}(u, P)<\mu_{0}\right\}, \mu_{0}>0$, and $A=G^{\prime}$.

$\left(A_{1}^{*}\right)$ Assume that, for any $a, b>0$, there is a constant $C>0$ such that

$$
G(u) \leq a, \quad\|u\|_{*} \leq b \Longrightarrow\|u\| \leq C,
$$

where $\|\cdot\|_{*}$ denotes another norm of $E$ such that $\|u\|_{*} \leq C\|u\|$ for all $u \in E$.

$$
\left(A_{2}^{*}\right) \text { Assume that } \lim _{u \in Y,\|u\| \rightarrow \infty} I(u)=-\infty, \sup _{Y} I:=\beta .
$$

If the even functional I satisfies (PS) condition at levelc for each $c \in[\gamma, \beta]$, then

$$
\mathscr{K}[\gamma-\epsilon, \beta+\epsilon] \cap(E \backslash(-P \cup P)) \neq \emptyset
$$

for all $\epsilon>0$ small, where $\left(\sup _{Y} I:=\beta(Y\right.$ and $M$ are two subspaces of $E$ with $\operatorname{dim} Y<\infty, \operatorname{dim} Y-\operatorname{codim} M \geq 1)$, $\inf _{Q^{* *}} I:=\gamma$, and $Q^{* *}:=Q^{*}(\rho) \cap I^{\beta}\left(Q^{*}(\rho):=\{u \in M:\right.$ $\left.\left(\|u\|_{*}^{p} /\|u\|^{2}\right)+\left(\left(\|u\|\|u\|_{*}\right) /\left(\|u\|+D_{*}\|u\|_{*}\right)\right)=\rho\right\}$, where $\rho>0$, $D_{*}>0$, and $p>2$ are fixed constants.

Now, we give an outline proof for our Theorem 9.

Proof of Theorem 9. Let $N_{k}$ denote the eigenspace of $\lambda_{k}$. We fix $k$ and let $E_{k}:=N_{1} \oplus \cdots \oplus N_{k}$. Consider another norm $\|\cdot\|_{*}:=\|\cdot\|_{p+1}$ of $E, p \in(1,((N+4) /(N-4)))$. Write $E=$ $E_{k-1} \oplus E_{k-1}^{\perp}$.

Let

$$
Q^{*}(\rho):=\left\{u \in E_{k-1}^{\perp}: \frac{\|u\|_{p+1}^{p+1}}{\|u\|^{2}}+\frac{\|u\|\|u\|_{p+1}}{\|u\|+D_{*}\|u\|_{p+1}}=\rho\right\},
$$

where $\rho, D_{*}$ are fixed constants. By our assumptions, we may find a constant $C>0$ such that

$$
F(x, t) \leq \frac{1}{4} \lambda_{1} t^{2}+C|t|^{p+1}, \forall x \in \Omega, \quad t \in R,
$$

where $1<p<(N+4) /(N-4)$. For any $a, b>0$, there is a constant $C>0$ such that

$$
I(u) \leq a, \quad\|u\|_{p+1} \leq b \Longrightarrow\|u\| \leq C .
$$

By Lemma 15,

$$
\lim _{u \in Y,\|u\| \rightarrow \infty} I(u)=-\infty
$$

where $Y=E_{k}$. Then $\left(A_{1}^{*}\right)$ and $\left(A_{2}^{*}\right)$ are satisfied. By Lemma 14, the condition $(A)$ holds.

Now, we define

$$
\sup _{Y} I:=\beta
$$

Let

$$
Q^{* *}:=Q^{*}(\rho) \cap I^{\beta}, \quad \inf _{Q^{* *}}:=\gamma
$$

By Lemma 12, I satisfies the (PS) condition. Thus, by Proposition 17 and the Proof of Theorem 5.7 in [9], we know that the functional $I$ posses a sequence sign-changing solution $\left\{u_{k}\right\}$.

\section{Conflict of Interests}

The authors declare that they have no competing interests.

\section{Acknowledgments}

The authors would like to thank the referees for valuable comments and suggestions in improving this paper. This work was supported by the National NSF (Grant no. 10671156) of China and Planned Projects for Postdoctoral Research Funds of Jiangsu Province (Grant no. 1301038C).

\section{References}

[1] A. C. Lazer and P. J. McKenna, "Large-amplitude periodic oscillations in suspension bridges: some new connections with nonlinear analysis," SIAM Review, vol. 32, no. 4, pp. 537-578, 1990.

[2] A. M. Micheletti and A. Pistoia, "Multiplicity results for a fourth-order semilinear elliptic problem," Nonlinear Analysis. Theory, Methods \& Applications A, vol. 31, no. 7, pp. 895-908, 1998. 
[3] M. Chipot, Variational Inequalities and Flow in Porous Media, vol. 52, Springer, New York, NY, USA, 1984.

[4] J. Zhang and S. Li, "Multiple nontrivial solutions for some fourth-order semilinear elliptic problems," Nonlinear Analysis. Theory, Methods \& Applications A, vol. 60, no. 2, pp. 221-230, 2005.

[5] Y. Wu, S. T. He, and Y. T. Shen, "Existence of nontrivial solutions to an asymptotically linear biharmonic equation," Acta Mathematica Sinica, vol. 54, no. 1, pp. 9-14, 2011.

[6] Adimurthi, M. Grossi, and S. Santra, "Optimal Hardy-Rellich inequalities, maximum principle and related eigenvalue problem," Journal of Functional Analysis, vol. 240, no. 1, pp. 36-83, 2006.

[7] X. Liu and Y. Huang, "On sign-changing solution for a fourthorder asymptotically linear elliptic problem," Nonlinear Analysis. Theory, Methods \& Applications A, vol. 72, no. 5, pp. 22712276, 2010.

[8] J. Zhou and X. Wu, "Sign-changing solutions for some fourthorder nonlinear elliptic problems," Journal of Mathematical Analysis and Applications, vol. 342, no. 1, pp. 542-558, 2008.

[9] W. Zou, Sign-Changing Critical Point Theory, Springer, New York, NY, USA, 2008.

[10] Z. Liu and J. Sun, "Invariant sets of descending flow in critical point theory with applications to nonlinear differential equations," Journal of Differential Equations, vol. 172, no. 2, pp. 257-299, 2001.

[11] A. Mao and Z. Zhang, "Sign-changing and multiple solutions of Kirchhoff type problems without the P.S. condition," Nonlinear Analysis. Theory, Methods \& Applications A, vol. 70, no. 3, pp. 1275-1287, 2009.

[12] H.-S. Zhou, "Existence of asymptotically linear Dirichlet problem," Nonlinear Analysis. Theory, Methods \& Applications A, vol. 44, pp. 909-918, 2001.

[13] Y. Liu and Z. Wang, "Biharmonic equations with asymptotically linear nonlinearities," Acta Mathematica Scientia B, vol. 27, no. 3, pp. 549-560, 2007. 


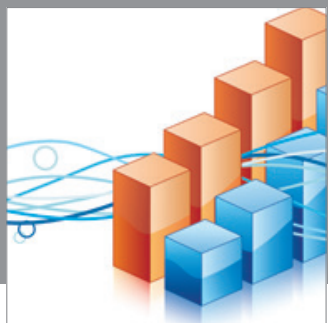

Advances in

Operations Research

mansans

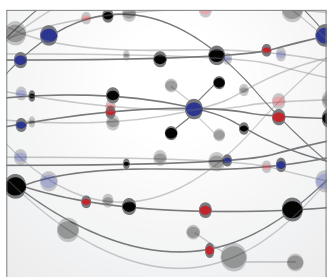

The Scientific World Journal
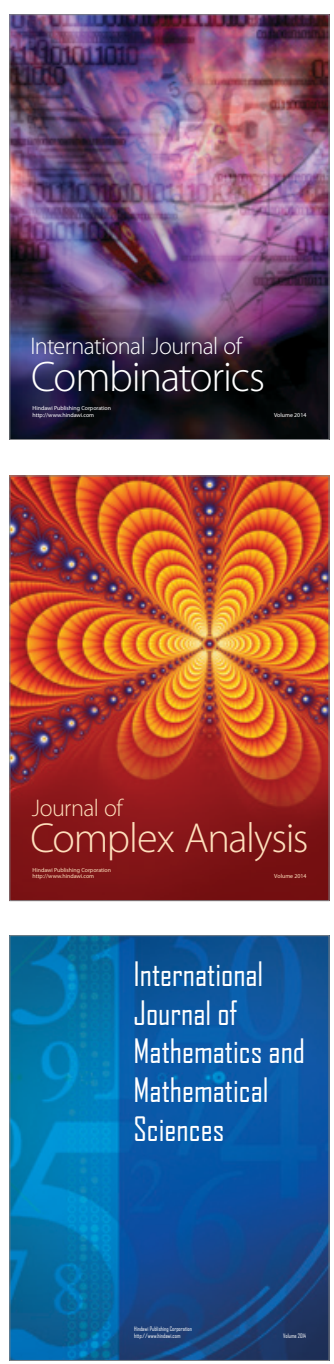
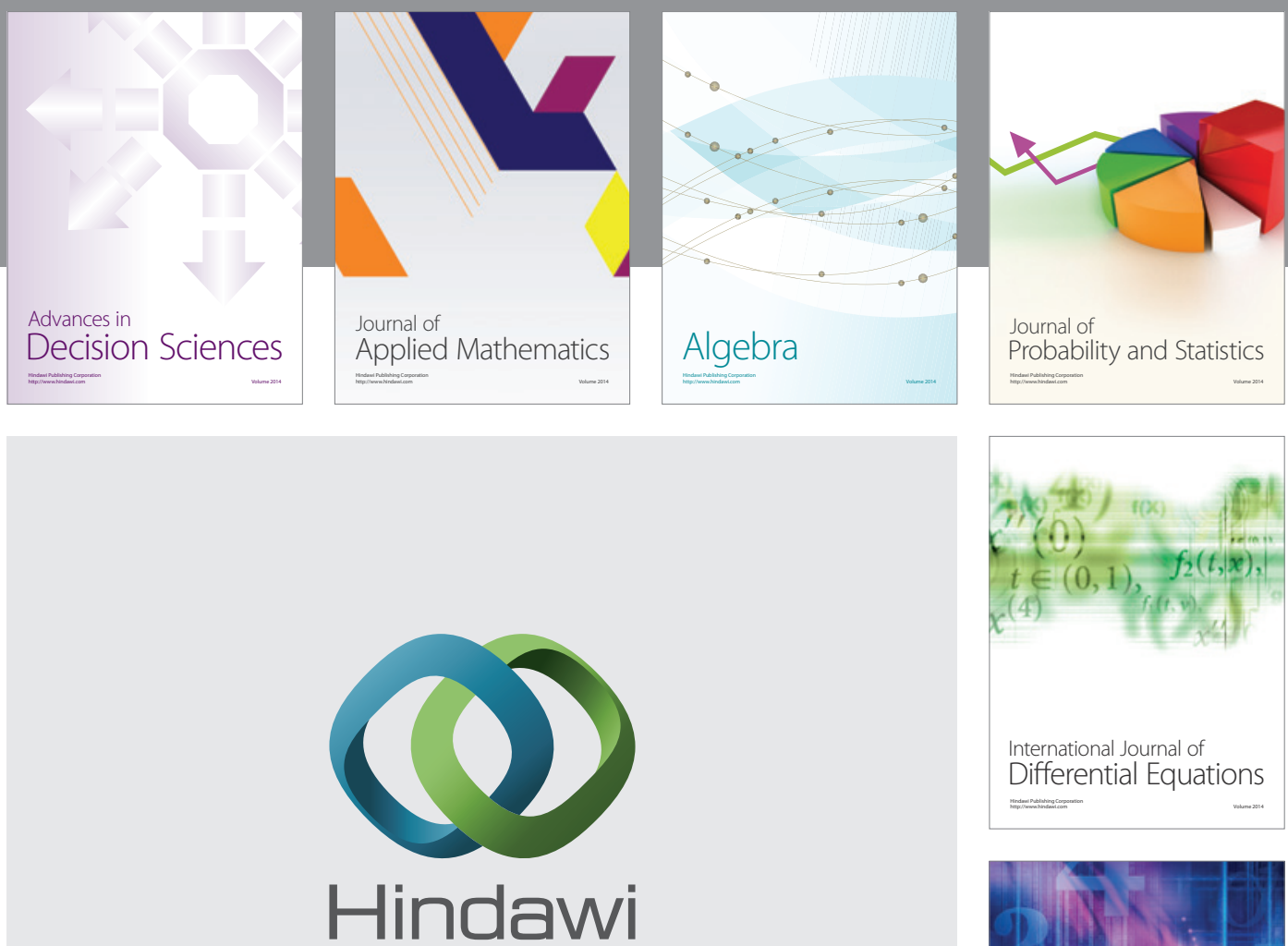

Submit your manuscripts at http://www.hindawi.com
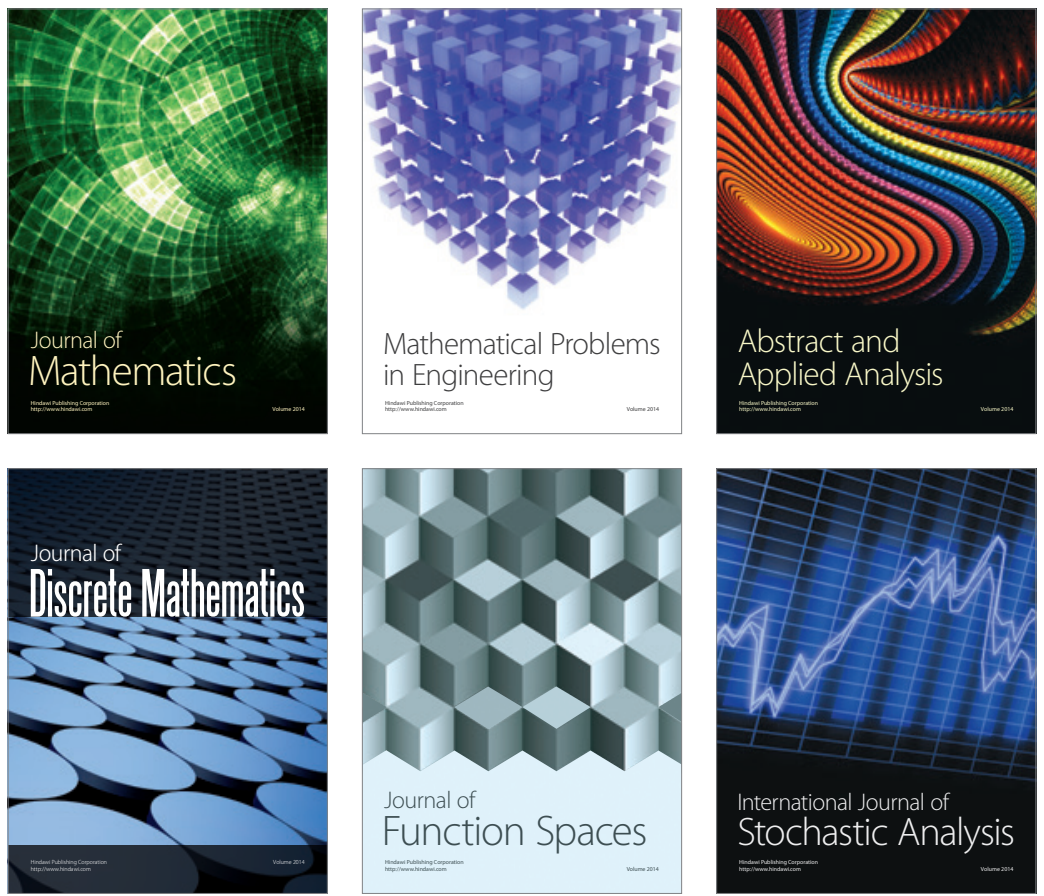

Journal of

Function Spaces

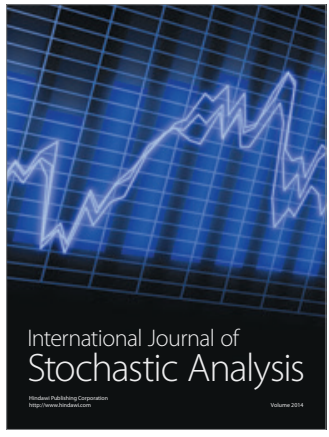

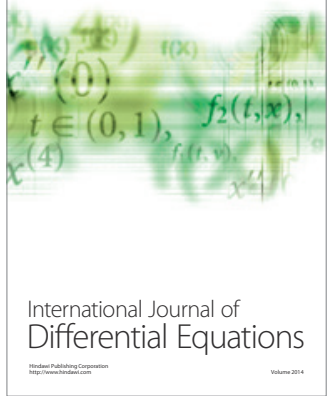
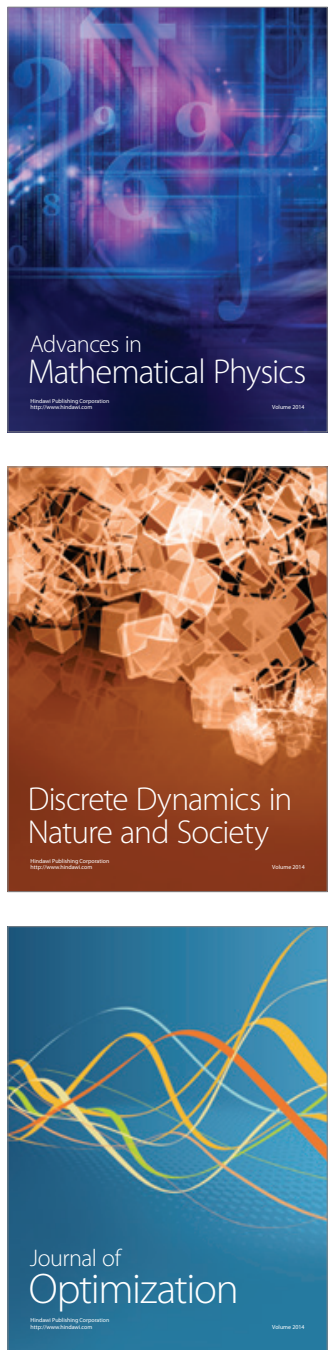